\title{
Bound States of a System of Two Fermions on Invariant Subspace
}

\author{
J. I. Abdullaev, A. M. Toshturdiev* \\ Samarkand State University, University Boulevard 15, Samarkand, Uzbekistan \\ Email: jabdullaev@mail.ru, ^atoshturdiyev@mail.ru
}

How to cite this paper: Abdullaev, J.I. and Toshturdiev, A.M. (2021) Bound States of a System of Two Fermions on Invariant Subspace. Journal of Modern Physics, 12, 35-49. https://doi.org/10.4236/jmp.2021.121004

Received: October 26, 2020

Accepted: January 11, 2021

Published: January 14, 2021

Copyright $\odot 2021$ by author(s) and Scientific Research Publishing Inc. This work is licensed under the Creative Commons Attribution International License (CC BY 4.0).

http://creativecommons.org/licenses/by/4.0/

\section{Abstract}

We consider a Hamiltonian of a system of two fermions on a three-dimensional lattice $\mathbb{Z}^{3}$ with special potential $\hat{v}$. The corresponding Shrödinger operator $H(\mathbf{k})$ of the system has an invariant subspace $L_{123}^{-}\left(\mathbb{T}^{3}\right)$, where we study the eigenvalues and eigenfunctions of its restriction $H_{123}^{-}(\mathbf{k})$. Moreover, there are shown that $H_{123}^{-}\left(k_{1}, k_{2}, \pi\right)$ has also infinitely many invariant subspaces $\mathfrak{R}_{123}^{-}(n), n \in \mathbb{N}$, where the eigenvalues and eigenfunctions of eigenvalue problem

$$
H\left(k_{1}, k_{2}, \pi\right) f=z f, f \in \mathfrak{R}_{123}^{-}(n)
$$

are explicitly found.

\section{Keywords}

Hamiltonian, Fermion, Bound State, Shrödinger Operator, Invariant Subspace, Total Quasi-Momentum, Eigenvalue, Birman-Schwinger Principle

\section{Introduction}

The nature of bound states of two-particle cluster operators for small parameter values was first studied in detail by Minlos and Mamatov [1] and then in a more general setting by Minlos and Mogilner [2]. In [3], Howland showed that the Rellich theorem on perturbations of eigenvalues does not extend to the resonance theory. Studying bound states of a two-particle system Hamiltonian $H$ on the $d$-dimensional lattice $\mathbb{Z}^{d}$ reduces to studying [2] [4] [5] [6] [7] the eigenvalues of a family of Shrödinger operators $H(\mathbf{k}), \mathbf{k} \in \mathbb{T}^{d}$, where $\mathbf{k}$ is the total quasi-momentum of a system. Moreover, eigenfunctions of $H(\mathbf{k})$ are interpreted as bound states of the Hamiltonian $H$, and eigenvalues, as the bound state 
energies. The bound states of $H$ of a system of two fermions on a one-dimensional lattice were studied in [4], a system of two bosons on a two-dimensional lattice was studied in [6], and perturbations of the eigenvalues of a two-particle Shrödinger operator on a one-dimensional lattice were studied in [8]. The finiteness of the number of eigenvalues of Shrödinger operator on a lattice was studied in the works [7] [9].

The discrete spectrum of the two-particle continuous Shrödinger operator

$$
h_{\lambda}=-\Delta+\lambda V
$$

was studied by many authors, with the conditions for the potential $V$ formulated in its coordinate representation. The condition for the finiteness of the set of negative elements of the spectrum and the absence of positive eigenvalues of $h_{\lambda}$ can be found in [10]. If $V \leq 0$, then the number of negative eigenvalues $N(\lambda)$ is a nondecreasing function of $\lambda \in(0, \infty)$, and each eigenvalue $z_{n}(\lambda)$ decreases on the half-axis $(0, \infty)$. It is known that when the coupling constant $\lambda$ decreases, the bound state energies of $h_{\lambda}$ tend to the boundary of the continuous spectrum (see [10]) and for some finite $\lambda$ are on the boundary. Two questions then arise: Does a bound or virtual state correspond to such a threshold state (i.e., is the corresponding wave function square-integrable)? And where do the bound states "disappear to" as $\lambda$ decreases further? The study of the first question was the subject in [11] [12]. Regarding the second question, it turns out that the bound state disappears by being absorbed into the continuous spectrum and becomes a resonance [5].

Here, we consider bound states of the Hamiltonian $\hat{H}$ (see (1)) of a system of two fermions on the three-dimensional lattice $\mathbb{Z}^{3}$ with the special potential $\hat{v}$ (see (5)). In other words, we study the discrete spectrum of a family of the Shrödinger operators $H(\mathbf{k}), \mathbf{k}=\left(k_{1}, k_{2}, k_{3}\right) \in \mathbb{T}^{3}$, (see (3)) corresponding to $\hat{H}$ in the invariant subspace $L_{123}^{-}\left(\mathbb{T}^{3}\right)$.

Restriction of the operator $H(\mathbf{k})$ in the invariant subspace $L_{123}^{-}\left(\mathbb{T}^{3}\right)$ is denoted by $H_{123}^{-}(\mathbf{k})$.

In the case $\mathbf{k}=\vec{\pi}:=(\pi, \pi, \pi)$, the operator $H(\vec{\pi})$ has an infinite number of eigenvalues of the form $6-\hat{v}(\mathbf{n}), \mathbf{n} \in \mathbb{Z}^{3}$ and the essential spectrum consists of the single point 6 . Here, the potential $\hat{v}$ is defined by (5) and $\bar{v}: \mathbb{N} \rightarrow \mathbb{R}$ is a decreasing function on $\mathbb{N}$ and $\bar{v} \in \ell_{2}(\mathbb{N})$. These eigenvalues

$z_{n}(\vec{\pi})=6-\bar{v}(n), n \in \mathbb{N}$ are arranged in ascending order,

$z_{1}(\vec{\pi})<\cdots<z_{n}(\vec{\pi})<\cdots$, and the smallest eigenvalue $z_{1}(\vec{\pi})=6-\bar{v}(1)$ is threefold, $z_{2}(\vec{\pi})=6-\bar{v}(2)$ is sevenfold, and the other eigenvalues

$z_{n}(\vec{\pi})=6-\bar{v}(n), n \geq 3$ are ninefold. All ninefold eigenvalues

$z_{n}(\vec{\pi})=6-\bar{v}(n), n \geq 3$ of the operator $H(\vec{\pi})$ are simple eigenvalues for the operator $H_{123}^{-}(\vec{\pi})$.

Further, we investigate eigenvalues and eigenfunctions of the restriction operator $H_{123}^{-}(\mathbf{k})$.

In the case $\mathbf{k}=\left(k_{1}, k_{2}, \pi\right)$ the corresponding operator $H_{123}^{-}\left(k_{1}, k_{2}, \pi\right)$ has infinitely many invariant subspaces $\mathfrak{R}_{123}^{-}(n):=L_{2}^{-}(\mathbb{T}) \otimes L_{2}^{-}(\mathbb{T}) \otimes L^{-}(n), n \in \mathbb{N}$. It 
is proved that the restriction $H_{123 n}^{-}\left(k_{1}, k_{2}, \pi\right)$ of the operator $H_{123}^{-}\left(k_{1}, k_{2}, \pi\right)$ in the invariant subspace $\mathfrak{R}_{123}^{-}(n)$ has no more than one eigenvalue. If exists, it can be calculated explicitly. For every $\left(k_{1}, k_{2}\right) \in(-\pi, \pi)^{2}$ the operator $H_{123}^{-}\left(k_{1}, k_{2}, \pi\right)$ has only a finite number of eigenvalues.

For any perturbation $\beta>0$, the essential spectrum $\{6\}$ of $H(\vec{\pi})$ becomes the essential spectrum $\sigma_{e s s}(H(\pi-2 \beta, \pi, \pi))=[6-2 \sin \beta, 6+2 \sin \beta]$. If the potential $\hat{v}$ is of the form (5), the Shrödinger equation $H_{123}^{-}(\pi-2 \beta, \pi, \pi) f=z f, f \in \mathfrak{R}_{123}^{-}(n)$ can be exactly solved (see Theorem 1 ).

The Shrödinger equations $H(\pi-2 \beta, \pi, \pi) f=z f$ and $H(\pi-2 \beta, \pi-2 \beta, \pi) f=z f, f \in \mathfrak{R}_{123}^{-}(n)$ with small $\beta$ are solved by using methods invariant subspaces and operator theory.

\section{Description of the Hamiltonian and Expansion in a Direct Integral}

The free Hamiltonian $\hat{H}_{0}$ of a system of two fermions on a three-dimensional lattice $\mathbb{Z}^{3}$ usually corresponds to a bounded self-adjoint operator acting in the Hilbert space $\ell_{2}^{a s}\left(\mathbb{Z}^{3} \times \mathbb{Z}^{3}\right):=\left\{f \in \ell_{2}\left(\mathbb{Z}^{3} \times \mathbb{Z}^{3}\right): f(\mathbf{x}, \mathbf{y})=-f(\mathbf{y}, \mathbf{x})\right\}$ by the formula

$$
\hat{H}_{0}=-\frac{1}{2 m} \Delta_{1}-\frac{1}{2 m} \Delta_{2}
$$

Here, $m$ is the fermion mass, which we assume to be equal to unity in what follows, $\Delta_{1}=\Delta \otimes I$ and $\Delta_{2}=I \otimes \Delta$, where $I$ is the identity operator, and the lattice Laplacian $\Delta$ is a difference operator that describes a translation of a particle from a side to a neighboring side,

$$
(\Delta \hat{\psi})(\mathbf{x})=\sum_{j=1}^{3}\left[\hat{\psi}\left(\mathbf{x}+\mathbf{e}_{j}\right)+\hat{\psi}\left(\mathbf{x}-\mathbf{e}_{j}\right)-2 \hat{\psi}(\mathbf{x})\right], \mathbf{x} \in \mathbb{Z}^{3}, \hat{\psi} \in \ell_{2}\left(\mathbb{Z}^{3}\right),
$$

where $\mathbf{e}_{1}=(1,0,0), \mathbf{e}_{2}=(0,1,0), \mathbf{e}_{3}=(0,0,1)$ are unit vectors in $\mathbb{Z}^{3}$. The total Hamiltonian $\hat{H}$ acts in the Hilbert space $\ell_{2}^{a s}\left(\mathbb{Z}^{3} \times \mathbb{Z}^{3}\right)$ and is the difference of the free Hamiltonian $\hat{H}_{0}$ and the interaction potential $\hat{V}_{2}$ of the two fermions (see [8] [13]):

$$
\hat{H}=\hat{H}_{0}-\hat{V}_{2}
$$

where

$$
\left(\hat{V}_{2} \hat{\psi}\right)(\mathbf{x}, \mathbf{y})=\hat{v}(\mathbf{x}-\mathbf{y}) \hat{\psi}(\mathbf{x}, \mathbf{y}), \quad \hat{\psi} \in \ell_{2}^{a s}\left(\left(\mathbb{Z}^{3}\right)^{2}\right):=\ell_{2}^{a s}\left(\mathbb{Z}^{3} \times \mathbb{Z}^{3}\right) .
$$

Hereafter, we assume that

$$
\hat{v} \in \ell_{2}\left(\mathbb{Z}^{3}\right) \text { and } \hat{v}(\mathbf{x})=\hat{v}(-\mathbf{x}) \geq 0 \quad \text { for all } \mathbf{x} \in \mathbb{Z}^{3}
$$

Under this condition, the Hamiltonian $\hat{H}$ is a bounded self-adjoint operator in $\ell_{2}^{a s}\left(\left(\mathbb{Z}^{3}\right)^{2}\right)$.

We pass to momentum representation using the Fourier transform [2] [4] [7]

$$
F: \ell_{2}^{a s}\left(\mathbb{Z}^{3} \times \mathbb{Z}^{3}\right) \rightarrow L_{2}^{a s}\left(\mathbb{T}^{3} \times \mathbb{T}^{3}\right)
$$


The Hamiltonian $H=H_{0}-V=F \hat{H} F^{-1}$ in the momentum representation commutes with the unitary operators $U_{\mathrm{s}}, \mathbf{s} \in \mathbb{Z}^{3}$, given by

$$
\left(U_{\mathbf{s}} f\right)\left(\mathbf{k}_{1}, \mathbf{k}_{2}\right)=\exp \left(-i\left(\mathbf{s}, \mathbf{k}_{1}+\mathbf{k}_{2}\right)\right) f\left(\mathbf{k}_{1}, \mathbf{k}_{2}\right), f \in L_{2}^{a s}\left(\mathbb{T}^{3} \times \mathbb{T}^{3}\right) .
$$

It follows that there exist decompositions of $L_{2}^{a s}\left(\mathbb{T}^{3} \times \mathbb{T}^{3}\right)$ and the operators $U_{\mathrm{s}}$ and $H$ into direct integrals (see [7] [9] and [10])

$$
L_{2}^{a s}\left(\mathbb{T}^{3} \times \mathbb{T}^{3}\right)=\int_{\mathbb{T}^{3}} \oplus L_{2}^{a s}\left(F_{\mathbf{k}}\right) \mathrm{d} \mathbf{k}, U_{\mathbf{s}}=\int_{\mathbb{T}^{3}} \oplus U_{\mathbf{s}}(\mathbf{k}) \mathrm{d} \mathbf{k}, H=\int_{\mathbb{T}^{3}} \oplus \tilde{H}(\mathbf{k}) \mathrm{d} \mathbf{k} .
$$

Here,

$$
F_{\mathbf{k}}=\left\{\left(\mathbf{k}_{1}, \mathbf{k}_{2}\right) \in \mathbb{T}^{3} \times \mathbb{T}^{3}: \mathbf{k}_{1}+\mathbf{k}_{2}=\mathbf{k}\right\}, \quad \mathbf{k} \in \mathbb{T}^{3},
$$

and $U_{\mathbf{s}}(\mathbf{k})$ is an operator of multiplication by the function $\exp (-i(\mathbf{s}, \mathbf{k}))$ in $L_{2}^{a s}\left(F_{\mathbf{k}}\right)$. The fiber operator $\tilde{H}(\mathbf{k})$ of $H$ also acts in $L_{2}^{a s}\left(F_{\mathbf{k}}\right)$ and is unitarly equivalent to $H(\mathbf{k}):=H_{0}(\mathbf{k})-V$, which is called the Shrödinger operator. This operator acts in the Hilbert space $L_{2}^{o}\left(\mathbb{T}^{3}\right):=\left\{f \in L_{2}\left(\mathbb{T}^{3}\right): f(-\mathbf{q})=-f(\mathbf{q})\right\}$ by the formula

$$
(H(\mathbf{k}) f)(\mathbf{q})=\varepsilon_{\mathbf{k}}(\mathbf{q}) f(\mathbf{q})-(2 \pi)^{-\frac{3}{2}} \int_{\mathbb{T}^{3}} v(\mathbf{q}-\mathbf{s}) f(\mathbf{s}) \mathrm{d} \mathbf{s} .
$$

The unperturbed operator $H_{0}(\mathbf{k})$ is an operator of multiplication by the function

$$
\begin{aligned}
\varepsilon_{\mathbf{k}}(\mathbf{q}) & =\varepsilon\left(\frac{\mathbf{k}}{2}+\mathbf{q}\right)+\varepsilon\left(\frac{\mathbf{k}}{2}-\mathbf{q}\right) \\
& =6-2 \cos \frac{k_{1}}{2} \cos q_{1}-2 \cos \frac{k_{2}}{2} \cos q_{2}-2 \cos \frac{k_{3}}{2} \cos q_{3} .
\end{aligned}
$$

From (3) and (4), it follows that

$$
H\left(k_{1}, k_{2}, k_{3}\right)=H\left(-k_{1}, k_{2}, k_{3}\right)=H\left(k_{1},-k_{2}, k_{3}\right)=H\left(k_{1}, k_{2},-k_{3}\right),
$$

so we can assume $k_{1}, k_{2}, k_{3} \in[0, \pi]$.

The perturbation operator $V$ is an integral operator in $L_{2}^{o}\left(\mathbb{T}^{3}\right)$ with the kernel

$$
(2 \pi)^{-\frac{3}{2}} v(\mathbf{q}-\mathbf{s})=(2 \pi)^{-\frac{3}{2}}(F \hat{v})(\mathbf{q}-\mathbf{s}),
$$

and belongs to the class of Hilbert-Schmidt operators $\Sigma_{2}$.

In this work, we consider the operator $H(\mathbf{k})$ with the potential $\hat{v}$ of the form

$$
\hat{v}(\mathbf{n})=\hat{v}\left(n_{1}, n_{2}, n_{3}\right)= \begin{cases}\bar{v}(|\mathbf{n}|), & \left|n_{1}\right|+\left|n_{2}\right| \leq 1 \\ 0, & \left|n_{1}\right|+\left|n_{2}\right| \geq 2\end{cases}
$$

where $|\mathbf{n}|=\left|n_{1}\right|+\left|n_{2}\right|+\left|n_{3}\right|$. Supporter is in the cylinder:

$$
D=\left\{\mathbf{n}=\left(n_{1}, n_{2}, n_{3}\right) \in \mathbb{Z}^{3}: n_{3} \in \mathbb{Z},\left|n_{1}\right|+\left|n_{2}\right| \leq 1\right\} .
$$

Since for every function $\hat{\psi} \in \ell_{2}^{a s}\left(\left(\mathbb{Z}^{3}\right)^{2}\right)$ the equality $\hat{\psi}(\mathbf{x}, \mathbf{x})=0, \mathbf{x} \in \mathbb{Z}^{3}$ holds, then the value of the potential $\hat{v}$ at the origin can be set arbitrary, since it does not affect the result, for simplicity, we assume that $\hat{v}(0)=0$. 
The function $\bar{v}: \mathbb{N} \rightarrow \mathbb{R}$ in (5) is decreasing in $\mathbb{N}$ i.e.,

$$
\bar{v}(1)>\bar{v}(2)>\cdots
$$

and belongs to $\ell_{2}(\mathbb{N})$. The kernel $v$, of the integral operator $V$, i.e., the Fourier transform $v(\mathbf{p})=(F \hat{v})(\mathbf{p})$, of the potential $\hat{v}$, has the form

$$
\begin{aligned}
& v(\mathbf{p}):=(F \hat{v})(\mathbf{p})=\frac{1}{(2 \pi)^{3 / 2}} \sum_{\mathbf{n} \in \mathbb{Z}^{3}} \hat{v}(\mathbf{n}) \mathrm{e}^{i(\mathbf{n}, \mathbf{p})} \\
& =\frac{1}{(2 \pi)^{3 / 2}}\left[2 \bar{v}(1)\left(\cos p_{1}+\cos p_{2}+\cos p_{3}\right)\right. \\
& \quad+2 \bar{v}(2)\left(\cos 2 p_{3}+2 \cos p_{1} \cos p_{2}+2 \cos p_{1} \cos p_{3}+2 \cos p_{2} \cos p_{3}\right) \\
& +2 \sum_{n=1}^{\infty} \bar{v}(n+2)\left(\cos (n+2) p_{3}+2 \cos (n+1) p_{3}\left(\cos p_{1}+\cos p_{2}\right)\right. \\
& \left.\left.+4 \cos p_{1} \cos p_{2} \cos n p_{3}\right)\right] .
\end{aligned}
$$

Eigenvalues of the operator $H(\mathbf{k})$. We note that the spectra of the operators $H_{0}(\mathbf{k})$ and $V$ are known. The operator $H_{0}(\mathbf{k})$ does not have eigenvalues, its spectrum is continuous and coincides with the range of the function $\varepsilon_{\mathbf{k}}$ :

$$
\sigma\left(H_{0}(\mathbf{k})\right)=[m(\mathbf{k}), M(\mathbf{k})], \text { where } m(\mathbf{k})=\min _{\mathbf{q} \in \mathbb{T}^{3}} \varepsilon_{\mathbf{k}}(\mathbf{q}), M(\mathbf{k})=\max _{\mathbf{q} \in \mathbb{T}^{3}} \varepsilon_{\mathbf{k}}(\mathbf{q}) .
$$

The spectrum of $V$ consists of the set $\{0, \bar{v}(n), n \in \mathbb{N}\}$. Under condition (2), the operator $V$ is a Hilbert-Schmidt operator and is hence compact. By the Weyl theorem [10], the essential spectrum of $H(\mathbf{k})$ coincides with the spectrum of $H_{0}(\mathbf{k})$ :

$$
\sigma_{e s s}(H(\mathbf{k}))=[m(\mathbf{k}), M(\mathbf{k})]
$$

If $\mathbf{k}=\vec{\pi}$, then the spectrum of $H(\vec{\pi})=6 I-V$ consists of eigenvalues of the form $6-\bar{v}(n), n \in \mathbb{N}$ and the essential spectrum is $\{6\}$. If $k_{j}=\pi$ (for some $j \in\{1,2,3\})$, then there exists a potential $\hat{v}$ such that $H(\mathbf{k})$ has an infinite number of eigenvalues outside the continuous spectrum (see [4] [14]).

We recall some notations and known facts. For any self-adjoint operator $B$ acting in a Hilbert space $\mathscr{C}$ without an essential spectrum to the right of $\mu \in \mathbb{R}$, we let $n(\mu, B)$ denote the number of its eigenvalues to the right of $\mu$. We let $N(\mathbf{k}, z)$ denote the number of eigenvalues of $H(\mathbf{k})$ to the left of $z \leq m(\mathbf{k})$, i.e., $N(\mathbf{k}, z)=n(-z,-H(\mathbf{k}))$. The number $N(\mathbf{k}, m(\mathbf{k}))$ in fact coincides with the number of eigenvalues outside the continuous spectrum of $H(\mathbf{k})$. It follows from the self-adjointness of $H(\mathbf{k})=H_{0}(\mathbf{k})-V$ and positivity of $V$ that

$$
\sigma(H(\mathbf{k})) \cap(M(\mathbf{k}), \infty)=\varnothing,
$$

and hence $\sigma_{\text {disc }}(H(\mathbf{k})) \subset(-\infty, m(\mathbf{k}))$. Therefore we seek only eigenvalues $z$ less than $m(\mathbf{k})$.

For any $\mathbf{k} \in \mathbb{T}^{3}$ and $z<m(\mathbf{k})$, we define the integral operator

$$
G(\mathbf{k}, z)=V^{\frac{1}{2}} r_{0}(\mathbf{k}, z) V^{\frac{1}{2}}
$$

where $r_{0}(\mathbf{k}, z)$ is the resolvent of the unperturbed operator $H_{0}(\mathbf{k})$. Under 
condition (2), the operator $V$ is positive, and we let $V^{\frac{1}{2}}$ denote the positive square root of the positive operator $V$. A solution $f$ of the Schrödinger equation

$$
H(\mathbf{k}) f=z f
$$

and the fixed points $\varphi$ of $G(\mathbf{k}, z)$ are connected by the relations

$$
f=r_{0}(\mathbf{k}, z) V^{\frac{1}{2}} \varphi \text { and } \varphi=V^{\frac{1}{2}} f .
$$

The following proposition (the Birman-Schwinger principle) holds [9].

Lemma 1. The number of eigenvalues of $H(\mathbf{k})$ to the left of $z<m(\mathbf{k})$ coincides with the number of eigenvalues of $G(\mathbf{k}, z)$ greater than unity, i.e., the equality

$$
N(\mathbf{k}, z)=n(1, G(\mathbf{k}, z))
$$

holds.

Lemma 2. If for some $\mathbf{k} \in \mathbb{T}^{3}$ the limit operator $\lim _{z \rightarrow m(\mathbf{k})^{-}} G(\mathbf{k}, z)=G(\mathbf{k}, m(\mathbf{k}))$ exists and is compact, then the equality

$$
N(\mathbf{k}, m(\mathbf{k}))=n(1, G(\mathbf{k}, m(\mathbf{k})))
$$

holds.

Equality (8) states that the number of eigenvalues of $H(\mathbf{k})$, to the left of $m(\mathbf{k})$ is equal to the number of eigenvalues of $G(\mathbf{k}, m(\mathbf{k}))$ greater than unity.

\section{Invariant Subspaces of $H(\mathrm{k})$}

In this section, we study the invariant subspaces with respect to the operator $H(\mathbf{k})$.

Let $L_{2}^{-}(\mathbb{T})=\left\{f \in L_{2}(\mathbb{T}): f(-p)=-f(p)\right\}$ be a subspace of the space $L_{2}(\mathbb{T})$, consisting of odd functions on $\mathbb{T}=[-\pi, \pi]$, and

$L_{2}^{+}(\mathbb{T})=\left\{f \in L_{2}(\mathbb{T}): f(-p)=f(p)\right\}$ be a subspace of $L_{2}(\mathbb{T})$, consisting of even functions on $\mathbb{T}$. In addition, we use the notation

$$
L_{123}^{-}\left(\mathbb{T}^{3}\right):=L_{2}^{-}(\mathbb{T}) \otimes L_{2}^{-}(\mathbb{T}) \otimes L_{2}^{-}(\mathbb{T}), \quad L_{123}^{+}\left(\mathbb{T}^{3}\right):=L_{2}^{+}(\mathbb{T}) \otimes L_{2}^{+}(\mathbb{T}) \otimes L_{2}^{+}(\mathbb{T}) .
$$

Note that $L_{123}^{-}\left(\mathbb{T}^{3}\right)$ is a subspace of the space $L_{2}^{o}\left(\mathbb{T}^{3}\right)$. It is natural to expect the invariance of the subspace $L_{123}^{-}\left(\mathbb{T}^{3}\right)$ with respect to the operator $H(\mathbf{k})$. It turns out that this subspace is invariant under the operator $H(\mathbf{k})$, i.e. the following statement holds.

Lemma 3. Let the potential $\hat{v}$ have the form (5). Then the subspace $L_{123}^{-}\left(\mathbb{T}^{3}\right)$ is invariant under the action of $H(\mathbf{k})$.

Proof. We prove that this subspace is invariant first with respect to $H_{0}(\mathbf{k})$, and then with respect to $V$. It follows from representation (4) that the function $\varepsilon_{\mathbf{k}}$ belongs to the subspace $L_{123}^{+}\left(\mathbb{T}^{3}\right)$, and it follows from the inclusion $f \in L_{123}^{-}\left(\mathbb{T}^{3}\right)$ that $\varepsilon_{\mathbf{k}} f \in L_{123}^{-}\left(\mathbb{T}^{3}\right)$. This proves that $L_{123}^{-}\left(\mathbb{T}^{3}\right)$ is invariant with respect to $H_{0}(\mathbf{k})$.

Simple calculations show that the function (see (7)) 


$$
(V f)\left(p_{1}, p_{2}, p_{3}\right)=\frac{1}{(2 \pi)^{\frac{3}{2}}} \int_{\mathbb{T}^{3}} v\left(p_{1}-s_{1}, p_{2}-s_{2}, p_{3}-s_{3}\right) f\left(s_{1}, s_{2}, s_{3}\right) \mathrm{d} s_{1} \mathrm{~d} s_{2} \mathrm{~d} s_{3}
$$

belongs to the subspace $L_{123}^{-}\left(\mathbb{T}^{3}\right)$ for $f \in L_{123}^{-}\left(\mathbb{T}^{3}\right)$. Hence, we prove the invariance of $L_{123}^{-}\left(\mathbb{T}^{3}\right)$ with respect to $V$, and it follows that $L_{123}^{-}\left(\mathbb{T}^{3}\right)$ is invariant with respect to $H(\mathbf{k})=H_{0}(\mathbf{k})-V$.

$H_{123}^{-}(\mathbf{k})$ denotes the restriction of $H(\mathbf{k})$ to the respective subspace $L_{123}^{-}\left(\mathbb{T}^{3}\right)$. The action of $H_{0(123)}^{-}(\mathbf{k}):=H_{0}(\mathbf{k})$ is unchanged, the unperturbed operator $H_{0}(\mathbf{k})$ is an operator of multiplication by the function $\varepsilon_{\mathbf{k}}$. We present the formula for $V_{123}^{-}=\left.V\right|_{L_{123}^{-}\left(\mathbb{T}^{3}\right)}$ operator $V$ acts on the element $f \in L_{123}^{-}\left(\mathbb{T}^{3}\right)$ according to the formula

$$
\left(V_{123}^{-} f\right)(\mathbf{p})=\frac{1}{\pi^{3}} \sum_{n=1}^{\infty} \bar{v}(n+2) \int_{\mathbb{T}^{3}} \sin p_{1} \sin p_{2} \sin n p_{3} \sin q_{1} \sin q_{2} \sin n q_{3} f(\mathbf{q}) \mathrm{d} \mathbf{q} .
$$

Note that for $\mathbf{k}=\vec{\pi}$, the spectrum of $H(\vec{\pi})=6 I-V$ consists only of the eigenvalues $6,6-\bar{v}(n), n \in \mathbb{N}$ and the essential spectrum $\{6\}$. Under condition (6) the number $z_{1}(\vec{\pi})=6-\bar{v}(1)$ is a threefold eigenvalue of $H(\vec{\pi})$, with the corresponding eigenfunctions

$$
\sin p_{1}, \sin p_{2}, \sin p_{3},
$$

the number $z_{2}(\vec{\pi})=6-\bar{v}(2)$ is a sevenfold eigenvalue with the corresponding eigenfunctions

$$
\begin{aligned}
& \sin 2 p_{3}, \cos p_{1} \sin p_{2}, \sin p_{1} \cos p_{2}, \cos p_{1} \sin p_{3}, \\
& \sin p_{1} \cos p_{3}, \cos p_{2} \sin p_{3}, \sin p_{2} \cos p_{3},
\end{aligned}
$$

for each $n \geq 3$, the number $z_{n}(\vec{\pi})=6-\bar{v}(n)$ is a ninefold eigenvalue, and the corresponding eigenfunctions are

$$
\begin{aligned}
& \sin (n+2) p_{3}, \sin p_{1} \cos (n+1) p_{3}, \sin p_{2} \cos (n+1) p_{3}, \\
& \sin (n+1) p_{3} \cos p_{1}, \sin (n+1) p_{3} \cos p_{2}, \sin n p_{3} \cos p_{1} \cos p_{2}, \\
& \sin p_{2} \cos p_{1} \cos n p_{3}, \sin p_{1} \cos p_{2} \cos n p_{3}, \sin p_{1} \sin p_{2} \sin n p_{3} .
\end{aligned}
$$

The number $z_{\infty}(\vec{\pi})=6$ is an eigenvalue of an infinite multiplicity, and the corresponding eigenfunctions are

$$
\psi_{\left(n_{1}, n_{2}, n_{3}\right)}^{---}(\mathbf{p})=\sin n_{1} p_{1} \sin n_{2} p_{2} \sin n_{3} p_{3}, n_{3} \in \mathbb{N}, n_{1}+n_{2} \geq 3 .
$$

All ninefold eigenvalues $z_{n}(\vec{\pi})=6-\bar{v}(n), n \geq 3$ of the operator $H(\vec{\pi})$ are simple eigenvalues for the operator $H_{123}^{-}(\vec{\pi})$, and the number $z_{\infty}(\vec{\pi})=6$ is an eigenvalue of an infinite multiplicity.

If the third coordinate $k_{3}$ of the total quasimomentum $\mathbf{k}$ is equal to $\pi$, then the operator $H\left(k_{1}, k_{2}, \pi\right)$ has infinitely many invariant subspaces $\mathfrak{R}_{123}^{-}(n), n \in \mathbb{N}$.

Next, we give a description of the invariant subspace $\mathfrak{R}_{123}^{-}(n), n \in \mathbb{N}$.

The system of functions

$$
\left\{\psi_{n}^{-}(q)=\frac{1}{\sqrt{\pi}} \sin n q\right\}_{n \in \mathbb{N}}
$$


is an orthonormal basis in the space $L_{2}^{-}(\mathbb{T})$. Let us denote by $L^{-}(n), n \in \mathbb{N}$ the one-dimensional subspace spanned by the vector $\psi_{n}^{-}$. The space $L_{2}^{-}(\mathbb{T})$ can be decomposed into the direct sum

$$
L_{2}^{-}(\mathbb{T})=\sum_{n=1}^{\infty} \oplus L^{-}(n) .
$$

This decomposition produces another decomposition

$$
\begin{aligned}
L_{123}^{-}\left(\mathbb{T}^{3}\right) & =\sum_{n=1}^{\infty} \oplus\left\{L_{2}^{-}(\mathbb{T}) \otimes L_{2}^{-}(\mathbb{T}) \otimes L^{-}(n)\right\} \\
& =\sum_{n=1}^{\infty} \oplus\left\{L_{12}^{-}\left(\mathbb{T}^{2}\right) \otimes L^{-}(n)\right\}=\sum_{n=1}^{\infty} \oplus \Re_{123}^{-}(n),
\end{aligned}
$$

where

$$
\mathfrak{R}_{123}^{-}(n):=L_{12}^{-}\left(\mathbb{T}^{2}\right) \otimes L^{-}(n), \quad L_{12}^{-}\left(\mathbb{T}^{2}\right)=L_{2}^{-}(\mathbb{T}) \otimes L_{2}^{-}(\mathbb{T}) .
$$

Lemma 4. Let the potential $\hat{v}$ have the form (5). Then the subspace $\mathfrak{R}_{123}^{-}(n)$ is invariant under $H_{123}^{-}\left(k_{1}, k_{2}, \pi\right)$ for any $n \in \mathbb{N}$.

Proof. Let $\left(f \psi_{n}^{-}\right)\left(p_{1}, p_{2}, p_{3}\right):=f\left(p_{1}, p_{2}\right) \psi_{n}^{-}\left(p_{3}\right)$, where $f \in L_{12}^{-}\left(\mathbb{T}^{2}\right)$, $\psi_{n}^{-} \in L^{-}(n)$ is an arbitrary element of $\mathfrak{R}_{123}^{-}(n)$. We consider the action of $H_{123}^{-}\left(k_{1}, k_{2}, \pi\right)=H_{0}\left(k_{1}, k_{2}, \pi\right)-V_{123}^{-}$on $f \psi_{n}^{-}$:

$$
\begin{aligned}
& \left(H_{0}\left(k_{1}, k_{2}, \pi\right) f \psi_{n}^{-}\right)(\mathbf{p}) \\
= & {\left[\left(6-2 \cos \frac{k_{1}}{2} \cos p_{1}-2 \cos \frac{k_{2}}{2} \cos p_{2}\right) f\left(p_{1}, p_{2}\right)\right] \psi_{n}^{-}\left(p_{3}\right), } \\
\left(V_{123}^{-} f \psi_{n}^{-}\right)(\mathbf{p}) & \\
= & {\left[\frac{\bar{v}(n+2)}{\pi^{2}} \int_{\mathbb{T}^{2}} \sin p_{1} \sin q_{1} \sin p_{2} \sin q_{2} f\left(q_{1}, q_{2}\right) \mathrm{d} q_{1} \mathrm{~d} q_{2}\right] \psi_{n}^{-}\left(p_{3}\right) . }
\end{aligned}
$$

To obtain the last formula (10), we use the orthogonality of the system of functions $\left\{\psi_{n}^{-}\right\}_{n \in \mathbb{N}}$ in $L_{2}^{-}(\mathbb{T})$. Relations (9) and (10) imply the equality

$$
\begin{aligned}
& \left(H_{123}^{-}\left(k_{1}, k_{2}, \pi\right) f \psi_{n}^{-}\right)\left(p_{1}, p_{2}, p_{3}\right) \\
& =\left(H_{0}\left(k_{1}, k_{2}, \pi\right) f \psi_{n}^{-}\right)\left(p_{1}, p_{2}, p_{3}\right)-\left(V_{123}^{-} f \psi_{n}^{-}\right)\left(p_{1}, p_{2}, p_{3}\right) \\
& =\left[\left(6-2 \cos \frac{k_{1}}{2} \cos p_{1}-2 \cos \frac{k_{2}}{2} \cos p_{2}\right) f\left(p_{1}, p_{2}\right)\right] \psi_{n}^{-}\left(p_{3}\right) \\
& -\left[\frac{\bar{v}(n+2)}{\pi^{2}} \int_{\mathbb{T}^{2}} \sin p_{1} \sin q_{1} \sin p_{2} \sin q_{2} f\left(q_{1}, q_{2}\right) \mathrm{d} q_{1} \mathrm{~d} q_{2}\right] \psi_{n}^{-}\left(p_{3}\right)
\end{aligned}
$$

which completes the proof of the lemma.

We denote by $H_{123 n}^{-}\left(k_{1}, k_{2}, \pi\right)$ restriction of the operator $H_{123}^{-}\left(k_{1}, k_{2}, \pi\right)$ in the invariant subspace $\mathfrak{R}_{123}^{-}(n)$. Formula (11) shows that the restriction $H_{123 n}^{-}\left(k_{1}, k_{2}, \pi\right)$ to the subspace $\Re_{123}^{-}(n)=L_{12}^{-}\left(\mathbb{T}^{2}\right) \otimes L^{-}(n)$ has the form

$$
H_{123 n}^{-}\left(k_{1}, k_{2}, \pi\right)=\left[2 I+H_{0}\left(k_{1}, k_{2}\right)-\bar{v}(n+2) V_{11}\right] \otimes I,
$$

where $I$ is the identity operator and $H_{123}^{(n)}(\mathbf{k}):=2 I+H_{0}(\mathbf{k})-\bar{v}(n+2) V_{11}$, $\mathbf{k}=\left(k_{1}, k_{2}\right)$, is a two-dimensional two-particle operator acting in $L_{12}^{-}\left(\mathbb{T}^{2}\right)$ by 
the formula

$$
\begin{aligned}
& \left(H_{123}^{(n)}(\mathbf{k}) f\right)(\mathbf{p}) \\
& =\left(2+\varepsilon_{\mathbf{k}}(\mathbf{p})\right) f(\mathbf{p})-\frac{\bar{v}(n+2)}{\pi^{2}} \int_{\mathbb{T}^{2}} \sin p_{1} \sin p_{2} \sin q_{1} \sin q_{2} f(\mathbf{q}) \mathrm{d} \mathbf{q},
\end{aligned}
$$

where $\varepsilon_{\mathbf{k}}(\mathbf{p})=4-2 \cos \frac{k_{1}}{2} \cos p_{1}-2 \cos \frac{k_{2}}{2} \cos p_{2}$, and $V_{11}$ is a one-dimensional integral operator in $L_{12}^{-}\left(\mathbb{T}^{2}\right)$ with the kernel

$$
v(\mathbf{p}, \mathbf{q})=\frac{1}{\pi^{2}} \sin p_{1} \sin p_{2} \sin q_{1} \sin q_{2} .
$$

Studying the eigenvalues of $H_{123 n}^{-}\left(k_{1}, k_{2}, \pi\right)$ by representations (12) reduces to studying the eigenvalues of

$$
H_{123}^{(n)}(\mathbf{k})=2 I+H_{0}(\mathbf{k})-\bar{v}(n+2) V_{11}, \mathbf{k}=\left(k_{1}, k_{2}\right)
$$

i.e. the three-dimensional problem reduces to the two-dimensional problem.

\section{Eigenvalues of the Operator $H_{123}^{-}(\mathrm{k})$}

Our main goal in this section is to study the behavior of the nondegenerate eigenvalue $z_{n+2}(\vec{\pi})=6-\bar{v}(n+2), n \in \mathbb{N}$ of $H_{123}^{-}(\vec{\pi})$ at small perturbations $\beta$ $\left(k_{1}=\pi-2 \beta\right.$ or $\left.k_{2}=\pi-2 \beta\right)$, i.e. the eigenvalues of $H_{123}^{-}(\pi-2 \beta, \pi, \pi)$ (or $\left.H_{123}^{-}(\pi, \pi-2 \beta, \pi)\right)$ at small perturbations $\beta$. The studying of the eigenvalues of $H_{123}^{-}(\pi-2 \beta, \pi, \pi)$ is reduced to study the eigenvalues of the operator $H_{123 n}^{-}(\pi-2 \beta, \pi, \pi)$ for each fixed $n \in \mathbb{N}$. In turn, the problem of studying the eigenvalues of the operator $H_{123 n}^{-}(\pi-2 \beta, \pi, \pi)$ by virtue of (12) is reduced to study of the discrete spectrum of the operator

$$
H_{123}^{(n)}(\pi-2 \beta, \pi)=2 I+H_{0}(\pi-2 \beta, \pi)-\bar{v}(n+2) V_{11} .
$$

Studying the eigenvalues of $H_{123}^{(n)}(\pi-2 \beta, \pi)$ and $H_{123}^{(n)}(\pi, \pi-2 \beta)$ reduces to studying the eigenvalues of $H_{\lambda}(k)$ acting in $L_{2}^{-}(\mathbb{T})$ by the formula

$$
\begin{aligned}
& \left(H_{\lambda}(k) f\right)(p)=\varepsilon_{k}(p) f(p)-\frac{\lambda}{\pi} \int_{\mathbb{T}} \sin p \sin q f(q) \mathrm{d} q, \\
& \varepsilon_{k}(p)=2-2 \cos \frac{k}{2} \cos p .
\end{aligned}
$$

It is known that the essential spectrum of

$H_{\lambda}(\pi-2 \beta)=H_{0}(\pi-2 \beta)-\lambda V_{1}, \beta \in\left(0, \frac{\pi}{2}\right]$ consists of a segment $[m(\beta), M(\beta)]$, where $m(\beta)=2-2 \sin \beta, M(\beta)=2+2 \sin \beta$.

Further we give some information about the eigenvalues and eigenfunctions of the operator $H_{\lambda}(k)$. Combining Theorem 6.3 in [6], Theorem 5.10 in [15] and Lemmas 1 and 2 we obtain the following statement about eigenvalues of the operator $H_{\lambda}(k)$.

Lemma 5. Let $\beta \in\left(0, \frac{\pi}{2}\right]$. 
a) If $\lambda<\sin \beta$, then the operator $H_{\lambda}(\pi-2 \beta)$ has no eigenvalues lying outside of the essential spectrum.

b) If $\lambda=\sin \beta$, then the left edge $m(\beta)$ of essential spectrum of the operator $H_{\lambda}(\pi-2 \beta)$ is a resonance.

c) If $\lambda>\sin \beta$, then the operator $H_{\lambda}(\pi-2 \beta)$ has a unique nondegenerate eigenvalue

$$
z_{\lambda}(\beta)=2-\lambda-\frac{1}{\lambda} \sin ^{2} \beta
$$

which lying in the left of the essential spectrum with corresponding normalized eigenfunction

$$
f_{\lambda}^{-}(p)=\frac{C_{\lambda} \sin p}{2-2 \sin \beta \cos p-z_{\lambda}(\beta)} \in L_{2}^{-}(\mathbb{T}) .
$$

Here $C_{\lambda}$ is the normalizing multiplicity.

d) The operator $H_{\lambda}(\pi-2 \beta)$ has no embedded eigenvalues in the interval $(m(\beta), M(\beta))$.

Hilbert space $L_{12}^{-}\left(\mathbb{T}^{2}\right)=L_{2}^{-}(\mathbb{T}) \otimes L_{2}^{-}(\mathbb{T})$ can be written as a direct sum:

$$
L_{2}^{-}(\mathbb{T}) \otimes L_{2}^{-}(\mathbb{T})=L_{2}^{-}(\mathbb{T}) \otimes L^{-}(1) \oplus\left(L_{2}^{-}(\mathbb{T}) \otimes L^{-}(1)\right)^{\perp} .
$$

The following lemma establishes a connection between the operators $H_{123}^{(n)}(\pi-2 \beta, \pi)$ and $H_{\lambda}(k)$.

Lemma 6. Let the potential $\hat{v}$ have the form (5). Then:

a) the subspace $L_{2}^{-}(\mathbb{T}) \otimes L^{-}(1)$ and its orthogonal complement $\left(L_{2}^{-}(\mathbb{T}) \otimes L^{-}(1)\right)^{\perp}$ are invariant under $H_{123}^{(n)}(\pi-2 \beta, \pi)$.

b) restriction of the operator $H_{123}^{(n)}(\pi-2 \beta, \pi)$ to the invariant subspace $\left(L_{2}^{-}(\mathbb{T}) \otimes L^{-}(1)\right)^{\perp}$ coinsides with the unperturbed operator $H_{0}(\pi-2 \beta, \pi)$.

c) restriction of the operator $H_{123}^{(n)}(\pi-2 \beta, \pi)$ to the invariant subspace $L_{2}^{-}(\mathbb{T}) \otimes L^{-}(1)$ can be represented as a tensor product:

$$
H_{123}^{(n)}(\pi-2 \beta, \pi)=\left[4 I+H_{0}(\pi-2 \beta)-\bar{v}(n+2) V_{1}\right] \otimes I .
$$

Here, $I$ is the identity operator, and $H_{\lambda(n)}(\pi-2 \beta):=H_{0}(\pi-2 \beta)-\lambda(n) V_{1}$, $\lambda(n)=\bar{v}(n+2)$ is a one-dimensional two-particle operator acting in $L_{2}^{-}(\mathbb{T})$ by the formula (13).

This lemma is proved in the same way as the Lemma 4 . In particular, part b) of the lemma implies that the operator $H_{123}^{(n)}(\pi-2 \beta, \pi)$ has no eigenfunctions in $\left(L_{2}^{-}(\mathbb{T}) \otimes L^{-}(1)\right)^{\perp}$. Thus, studying the eigenvalues of the operator $H_{123}^{(n)}(\pi-2 \beta, \pi)$ is reduced to studying eigenvalues of the operator $H_{\lambda(n)}(\pi-2 \beta)=H_{0}(\pi-2 \beta)-\lambda(n) V_{1}$.

From Lemmas 5 - 6 and tensor product (15) implies the following statement regarding operator $H_{123}^{(n)}(\pi-2 \beta, \pi)$.

Theorem 1. Let $\beta \in\left(0, \frac{\pi}{2}\right]$ and $n \in \mathbb{N}$.

a) If $\bar{v}(n+2)<\sin \beta$, then the operator $H_{123}^{(n)}(\pi-2 \beta, \pi)$ has no eigenvalues lying outside of the essential spectrum. 
b) If $\bar{v}(n+2)=\sin \beta$, then the left edge $m(\beta)$ of essential spectrum of the operator $H_{123}^{(n)}(\pi-2 \beta, \pi)$ is a resonance.

c) If $\bar{v}(n+2)>\sin \beta$, then the operator $H_{123}^{(n)}(\pi-2 \beta, \pi)$ has a unique nondegenerate eigenvalue

$$
z_{123}^{(n)}(\pi-2 \beta, \pi)=4+z_{\lambda(n)}(\beta)=6-\bar{v}(n+2)-\frac{1}{\bar{v}(n+2)} \sin ^{2} \beta,
$$

which lies in the left of the essential spectrum and with the corresponding normalized eigenfunction

$$
f_{\lambda(n)}^{--}\left(p_{1}, p_{2}\right)=f_{\lambda(n)}^{-}\left(p_{1}\right) \frac{\sin p_{2}}{\sqrt{\pi}}=f_{\lambda(n)}^{-}\left(p_{1}\right) \psi_{1}^{-}\left(p_{2}\right) \in L_{2}^{-}(\mathbb{T}) \otimes L^{-}(1),
$$

where $f_{\lambda(n)}^{-}$is the normalized eigenfunction of the operator $H_{\lambda(n)}(\pi-2 \beta)$ corresponding to the eigenvalue $z_{\lambda(n)}(\beta)$, the operator $H_{\lambda(n)}(k)$ is defined by the formula (13).

d) The operator $H_{123}^{(n)}(\pi-2 \beta, \pi)$ has no embedded eigenvalues in the interval $(m(\beta), M(\beta))$.

Similar statement is true for the operator $H_{123}^{(n)}(\pi, \pi-2 \beta)$. The eigenvalues of the operators $H_{123}^{(n)}(\pi, \pi-2 \beta)$ and $H_{123}^{(n)}(\pi-2 \beta, \pi)$ are same, but eigenfunctions differ with variable replacement $p_{1}$ and $p_{2}$. In other words, the operators $H_{123}^{(n)}\left(k_{1}, k_{2}\right)$ and $H_{123}^{(n)}\left(k_{2}, k_{1}\right)$ are unitary equivalent. Therefore, the operators $H_{123 n}^{-}\left(k_{1}, k_{2}, \pi\right)$ and $H_{123 n}^{-}\left(k_{2}, k_{1}, \pi\right)$ are unitary equivalent too.

Similar statement can relatively be formulated for the operator $H_{123}^{(n)}(\pi-2 \beta, \pi-2 \beta)$. For this purpose, we introduce the following notation. Through

$$
\Delta_{n}(\beta, z)=1-\frac{\bar{v}(n+2)}{\pi^{2}} \int_{\mathbb{T}^{2}} \frac{\sin ^{2} p_{1} \sin ^{2} p_{2} \mathrm{~d} p_{1} \mathrm{~d} p_{2}}{2+2\left(2-\sin \beta \cos p_{1}-\sin \beta \cos p_{2}\right)-z}
$$

we denote the Fredholm determinant of the operator $I-\bar{v}(n+2) V_{11} r_{0}(\beta, z)$, where $r_{0}(\beta, z)$ is the resolvent of the operator $2 I+H_{0}(\pi-2 \beta, \pi-2 \beta)$, and $V_{11}$ is an integral operator with the kernel

$$
v(\mathbf{p}, \mathbf{q})=\frac{1}{\pi^{2}} \sin p_{1} \sin p_{2} \sin q_{1} \sin q_{2} .
$$

Through $C_{11}^{--}$denote the value of the following integral:

$$
C_{11}^{--}=\frac{1}{\pi^{2}} \int_{\mathbb{T}^{2}} \frac{\sin ^{2} p_{1} \sin ^{2} p_{2} \mathrm{~d} p_{1} \mathrm{~d} p_{2}}{2\left(2-\cos p_{1}-\cos p_{2}\right)}=\int_{\mathbb{T}^{2}} \frac{\left|\psi_{1}^{-}\left(p_{1}\right)\right|^{2}\left|\psi_{1}^{-}\left(p_{2}\right)\right|^{2} \mathrm{~d} p_{1} \mathrm{~d} p_{2}}{2 \varepsilon(\mathbf{p})} .
$$

Simple calculations reveal the following approximate value $C_{11}^{--} \approx 0.302347$.

Theorem 2. Let $\beta \in\left(0, \frac{\pi}{2}\right], n \in \mathbb{N}$.

a) If $\bar{v}(n+2)<\frac{\sin \beta}{C_{11}^{--}}$, then the operator $H_{123}^{(n)}(\pi-2 \beta, \pi-2 \beta)$ has no eigenvalues lying outside of the essential spectrum.

b) If $\bar{v}(n+2)=\frac{\sin \beta}{C_{11}^{--}}$, then the left edge $m(\beta)=6-4 \sin \beta$ of the spectrum 
of the operator $H_{123}^{(n)}(\pi-2 \beta, \pi-2 \beta)$ is an eigenvalue.

c) If $\bar{v}(n+2)>\frac{\sin \beta}{C_{11}^{--}}$, then the operator $H_{123}^{(n)}(\pi-2 \beta, \pi-2 \beta)$ has a unique nondegenerate eigenvalue $z_{123}^{(n)}(\pi-2 \beta, \pi-2 \beta)$ below the essential spectrum.

d) The operator $H_{123}^{(n)}(\pi-2 \beta, \pi-2 \beta)$ has no embedded eigenvalues in the interval $(m(\beta), M(\beta))$.

This theorem is proved in similar way as Lemma 5 . There are some differences:

1) In the Theorem 2 , the eigenvalue $z_{123}^{(n)}(\pi-2 \beta, \pi-2 \beta)$ was calculated with the accuracy of $\beta^{2}$ :

$$
z_{123}^{(n)}(\pi-2 \beta, \pi-2 \beta)=6-\bar{v}(n+2)-\frac{2}{\bar{v}(n+2)} \sin ^{2} \beta+O\left(\beta^{4}\right)
$$

and corresponding normalized eigenfunction has the form

$$
\begin{aligned}
& f_{123}^{(n)}\left(p_{1}, p_{2}\right) \\
& =\frac{C_{n}(\beta) \sin p_{1} \sin p_{2}}{6-2 \sin \beta \cos p_{1}-2 \sin \beta \cos p_{2}-z_{123}^{(n)}(\pi-2 \beta, \pi-2 \beta)} \in L_{12}^{-}\left(\mathbb{T}^{2}\right),
\end{aligned}
$$

where $C_{n}(\beta)$ is the normalizing multiplicity.

2) Left edge $m(\beta)=6-2 \sin \beta$ of the essential spectrum is a resonance for the operator $H_{123}^{(n)}(\pi-2 \beta, \pi)$, but for the operator $H_{123}^{(n)}(\pi-2 \beta, \pi-2 \beta)$ the left edge $m(\beta)=6-4 \sin \beta$ of the essential spectrum is the eigenvalue, i.e. the equation $H_{123}^{(n)}(\pi-2 \beta, \pi-2 \beta) f=m(\beta) f$ has a non-trivial solution

$$
f\left(p_{1}, p_{2}\right)=\frac{C \sin p_{1} \sin p_{2}}{2-\cos p_{1}-\cos p_{2}}
$$

and it belongs to $L_{12}^{-}\left(\mathbb{T}^{2}\right)$.

\section{Conclusions}

1) We have shown that the operator $H_{123}^{-}\left(k_{1}, k_{2}, \pi\right)$ has infinitely many invariant subspaces $\mathfrak{R}_{123}^{-}(n), n \in \mathbb{N}$. It has been proved that if condition $\bar{v}(n+2)>\sin \beta$ holds then the operator $H_{123 n}^{-}(\pi-2 \beta, \pi, \pi)$ has a unique simple eigenvalue $z_{123}^{(n)}(\pi-2 \beta, \pi)$ of the form (16), otherwise, the operator has no eigenvalues outside of the essential spectrum. A similar statement holds for the operator $H_{123 n}^{-}(\pi-2 \beta, \pi-2 \beta, \pi)$.

2) Without loss of generality it can be assumed that $\bar{v}(3) \leq 1$. Since, if $\bar{v}(3)>1$ then it follows from $\lim _{n \rightarrow \infty} \bar{v}(n)=0$ that there exists a number $m \in \mathbb{N}$ such that $\bar{v}(m+2) \leq 1$ and monotonicity of $\bar{v}$ implies that $\bar{v}(n)>1$ for $n=3,4, \cdots, m+1$, and in this case, the eigenvalues $z_{123}^{(n)}(\pi-2 \beta, \pi), n=1,2, \cdots, m-1$ of $H_{123}^{-}(\pi-2 \beta, \pi, \pi)$ exist for all $\beta \in[0, \pi / 2]$.

For a fixed $\beta \in(0, \pi / 2]$ there exists $m \in \mathbb{N}$ such that $\sin \beta \in(\bar{v}(m+3), \bar{v}(m+2))$ and the operator $H_{123}^{-}(\pi-2 \beta, \pi, \pi)$ has $m$ nondegenerate eigenvalues outside of the essential spectrum (see Theorem 1 ): 


$$
\begin{gathered}
z_{123}^{(1)}(\pi-2 \beta, \pi, \pi):=z_{123}^{(1)}(\pi-2 \beta, \pi)=6-\bar{v}(3)-\frac{1}{\bar{v}(3)} \sin ^{2} \beta, \\
z_{123}^{(2)}(\pi-2 \beta, \pi, \pi):=z_{123}^{(2)}(\pi-2 \beta, \pi)=6-\bar{v}(4)-\frac{1}{\bar{v}(4)} \sin ^{2} \beta, \\
\vdots \\
z_{123}^{(m)}(\pi-2 \beta, \pi, \pi):=z_{123}^{(m)}(\pi-2 \beta, \pi)=6-\bar{v}(m+2)-\frac{1}{\bar{v}(m+2)} \sin ^{2} \beta .
\end{gathered}
$$

The corresponding normalized eigenfunctions are of the forms:

$$
\begin{gathered}
f_{123 \lambda(1)}^{---}\left(p_{1}, p_{2}, p_{3}\right)=f_{\lambda(1)}^{-}\left(p_{1}\right) \psi_{1}^{-}\left(p_{2}\right) \psi_{1}^{-}\left(p_{3}\right) \in L_{2}^{-}(\mathbb{T}) \otimes L^{-}(1) \otimes L^{-}(1) \\
f_{123 \lambda(2)}^{---}\left(p_{1}, p_{2}, p_{3}\right)=f_{\lambda(2)}^{-}\left(p_{1}\right) \psi_{1}^{-}\left(p_{2}\right) \psi_{2}^{-}\left(p_{3}\right) \in L_{2}^{-}(\mathbb{T}) \otimes L^{-}(1) \otimes L^{-}(2), \\
\vdots \\
f_{123 \lambda(m)}^{---}\left(p_{1}, p_{2}, p_{3}\right)=f_{\lambda(m)}^{-}\left(p_{1}\right) \psi_{1}^{-}\left(p_{2}\right) \psi_{m}^{-}\left(p_{3}\right) \in L_{2}^{-}(\mathbb{T}) \otimes L^{-}(1) \otimes L^{-}(m),
\end{gathered}
$$

where, $f_{\lambda(m)}^{-}$is the normalized eigenfunction of the operator $H_{\lambda(m)}(\pi-2 \beta)$ corresponding to the eigenvalue $z_{\lambda(m)}(\beta)$ and the operator $H_{\lambda(m)}(k)$ is defined by the formula (13), $\lambda(m)=\bar{v}(m+2)$.

The eigenvalues of the operators $H_{123}^{-}(\pi-2 \beta, \pi, \pi)$ and $H_{123}^{-}(\pi, \pi-2 \beta, \pi)$ are same but eigenfunctions differ with variable replacement $p_{1}$ and $p_{2}$. In other words, the operators $H_{123}^{-}(\pi-2 \beta, \pi, \pi)$ and $H_{123}^{-}(\pi, \pi-2 \beta, \pi)$ are unitary equivalent.

In the case $\sin \beta=\bar{v}(m+2)$, the left edge $m(\beta)=6-2 \sin \beta$ of the essential spectrum is a resonance of the operator $H_{123}^{-}(\pi-2 \beta, \pi, \pi)$ (see Theorem 1 ).

3) Let for some $m \in \mathbb{N}$ the relation $\sin \beta \in\left(\bar{v}(m+3) C_{11}^{--}, \bar{v}(m+2) C_{11}^{--}\right)$ hold then the operator $H_{123}^{-}(\pi-2 \beta, \pi-2 \beta, \pi)$ has $m$ nondegenerate eigenvalues outside the essential spectrum (see Theorem 2) and for small $\beta$ :

$$
\begin{aligned}
& z_{123}^{(1)}(\pi-2 \beta, \pi-2 \beta, \pi):=z_{123}^{(1)}(\pi-2 \beta, \pi-2 \beta) \\
& =6-\bar{v}(3)-\frac{2}{\bar{v}(3)} \sin ^{2} \beta+O\left(\beta^{4}\right), \\
& z_{123}^{(2)}(\pi-2 \beta, \pi-2 \beta, \pi):=z_{123}^{(2)}(\pi-2 \beta, \pi-2 \beta) \\
& =6-\bar{v}(4)-\frac{2}{\bar{v}(4)} \sin ^{2} \beta+O\left(\beta^{4}\right), \\
& \quad \vdots \\
& z_{123}^{(m)}(\pi-2 \beta, \pi-2 \beta, \pi):=z_{123}^{(m)}(\pi-2 \beta, \pi-2 \beta) \\
& =6-\bar{v}(m+2)-\frac{2}{\bar{v}(m+2)} \sin ^{2} \beta+O\left(\beta^{4}\right) .
\end{aligned}
$$

The corresponding normalized eigenfunctions are of the forms:

$$
\begin{aligned}
& f_{123}^{(1)-}\left(p_{1}, p_{2}, p_{3}\right)=f_{123}^{(1)}\left(p_{1}, p_{2}\right) \psi_{1}^{-}\left(p_{3}\right) \in L_{12}^{-}\left(\mathbb{T}^{2}\right) \otimes L^{-}(1), \\
& f_{123}^{(2)-}\left(p_{1}, p_{2}, p_{3}\right)=f_{123}^{(2)}\left(p_{1}, p_{2}\right) \psi_{2}^{-}\left(p_{3}\right) \in L_{12}^{-}\left(\mathbb{T}^{2}\right) \otimes L^{-}(2),
\end{aligned}
$$




$$
f_{123}^{(m)-}\left(p_{1}, p_{2}, p_{3}\right)=f_{123}^{(m)}\left(p_{1}, p_{2}\right) \psi_{m}^{-}\left(p_{3}\right) \in L_{12}^{-}\left(\mathbb{T}^{2}\right) \otimes L^{-}(m),
$$

where, $f_{123}^{(m)}$ is the normalized eigenfunction of the operator $H_{123}^{(m)}(\pi-2 \beta, \pi-2 \beta)$ corresponding to the eigenvalue $z_{123}^{(m)}(\pi-2 \beta, \pi-2 \beta)$ defined by the formula (17).

In the case $\sin \beta=\bar{v}(m+2) C_{11}^{--}$, the left edge $m(\beta)=6-4 \sin \beta$ of the essential spectrum is the eigenvalue of $H_{123}^{-}(\pi-2 \beta, \pi-2 \beta, \pi)$ (see Theorem 2) with the corresponding eigenfunction

$$
f(\mathbf{p})=\frac{C \sin p_{1} \sin p_{2}}{2-\cos p_{1}-\cos p_{2}} \cdot \sin m p_{3} \in L_{12}^{-}\left(\mathbb{T}^{2}\right) \otimes L^{-}(m) .
$$

Remark 1. If the potential $\hat{v}$ is even in all arguments $p_{1}, p_{2}, p_{3}$ and the condition $\hat{v} \in \ell_{2}\left(\mathbb{Z}^{3}\right)$ holds, then the statements of Lemmas 3 - 4 remain valid.

Remark 2. If $k_{3} \neq \pi$, then the subspaces $\mathfrak{R}_{123}^{-}(n), n \in \mathbb{N}$ are not invariant under the operator $H_{123}^{-}\left(k_{1}, k_{2}, k_{3}\right)$.

\section{Acknowledgements}

This work was supported by the Grant OT-F4-66 of Fundamental Science Foundation of Uzbekistan.

\section{Conflicts of Interest}

The authors declare no conflicts of interest regarding the publication of this paper.

\section{References}

[1] Mamatov, Sh.S. and Minlos, R.A. (1989) Theoretical and Mathematical Physics, 79, 455-466. https://doi.org/10.1007/BF01016525

[2] Minlos, R.A. and Mogilner, A.I. (1989) Some Problems Concerning Spectra of Lattice Models. In: Exner, P. and Seba, P., Eds., Schrödinger Operators. Standard and Nonstandard, World. Scientific, Singapore, 243-257.

[3] Howland, J.S. (1974) Pacific Journal of Mathematics, 55, 157-176. https://doi.org/10.2140/pjm.1974.55.157

[4] Abdullaev, J.I. (2006) Theoretical and Mathematical Physics, 147, 486-495. https://doi.org/10.1007/s11232-006-0055-Z

[5] Rauch, J. (1980) Journal of Functional Analysis, 35, 304-315. https://doi.org/10.1016/0022-1236(80)90085-3

[6] Abdullaev, J.I. and Kuliev, K.D. (2016) Theoretical and Mathematical Physics, 186, 231-250. https://doi.org/10.1134/S0040577916020082

[7] Muminov, M.I. and Ghoshal, S.K. (2020) Complex Analysis and Operator Theory, 14, Article No. 11. https://doi.org/10.1007/s11785-019-00978-Z

[8] Abdullaev, J.I. (2005) Theoretical and Mathematical Physics, 145, 1551-1558. https://doi.org/10.1007/s11232-005-0182-y

[9] Abdullaev, J.I. and Ikromov, I.A. (2007) Theoretical and Mathematical Physics, 152, 1299-1312. https://doi.org/10.1007/s11232-007-0114-0 
[10] Reed, M. and Simon, B. (1978) Methods of Modern Mathematical Physics Ser.: Analysis of Operators.

[11] Simon, B. (1976) Annals of Physics, 97, 279-288.

https://doi.org/10.1016/0003-4916(76)90038-5

[12] Klaus, M. (1977) Annals of Physics, 108, 288-300. https://doi.org/10.1016/0003-4916(77)90015-X

[13] Faria da Viega, P.A., Ioriatti, L. and O'Carrol, M. (2002) Physical Review E, 66, Article ID: 016130. https://doi.org/10.1103/PhysRevE.66.016130

[14] Abdullaev, J.I. (2005) Uzbek Mathematical Journal, No. 1, 3-11.

[15] Ando, K., Isozaki, H. and Morioka, H. (2016) Annales Henri Poincaré, 17, 2103-2171. https://doi.org/10.1007/s00023-015-0430-0 